\title{
The structure of spiral galaxies: radial profiles in stellar mass-to-light ratio and the dark matter distribution
}

\author{
L. Portinari ${ }^{1}$ and P. Salucci ${ }^{2}$ \\ 1 Tuorla Observatory, Department of Physics and Astronomy, University of Turku, Väisäläntie 20, 21500 Piikkiö, Finland \\ e-mail: lporti@utu.fi \\ 2 SISSA/ISAS, via Beirut 4, 34014 Trieste, Italy \\ e-mail: salucci@sissa.it
}

Received 28 November 2008 / Accepted 21 April 2009

\begin{abstract}
Context. The colour and metallicity gradients observed in spiral galaxies suggest that the mass-to-light ratio $\left(M_{\star} / L\right)$ of the stellar disc is a function of radius. This is indeed predicted by chemo-photometric models of galactic discs.

Aims. We investigate the distribution of luminous and dark matter in spiral galaxies, taking into account the radial dependence of the stellar $M_{\star} / L$, which is usually assumed to be constant in studies of the mass structure.

Methods. From earlier chemo-photometric models and in agreement with the observed radial profiles of galaxy colours, we derive the typical average $M_{\star} / L$ profile of the stellar discs of spiral galaxies. We computed the corresponding variable mass-to-light (VML) stellar surface density profile and then the VML disc contribution to the circular velocity. We used the latter, combined with a wellstudied dark matter velocity profile, to mass model co-added rotation curves.

Results. By investigating rotation curves in the framework of VML stellar discs, we confirm the scenario obtained with the constant $M_{\star} / L$ assumption: a dark matter halo with a shallow core, an inner baryon-dominated region, and a larger proportion of dark matter in smaller objects. However, the resulting size of the the dark halo core and of the inner baryon dominance region are somewhat smaller. The stronger role that VML discs have in the innermost regions is important for constraining the galaxy mass structure in both $\Lambda$ Cold Dark Matter and MOND scenarios.
\end{abstract}

Key words. galaxies: evolution - galaxies: spiral - dark matter

\section{Introduction}

Within Newtonian gravity the mass model of spiral galaxies, including an (assumed spherical) dark halo, can be derived from high-quality rotation curves with little presence of non circular motions due to bars, spiral structure or oval distortions; or from co-added rotation curves (e.g. Broeils 1992; Salucci \& Burkert $(2000)^{1}$. A careful account of the distribution of the baryonic matter is crucial. This includes the contribution of the stellar and $\mathrm{HI}$ discs, and of a significant central bulge in early type spiral galaxies (not investigated here).

The surface density of the stellar disc $\Sigma_{\star}(R)$ is obtained from the surface brightness $I(R)$, often fitted with an exponential profile of scalelength $h$ (Freeman 1970)

$I(R)=I_{0} \mathrm{e}^{-R / h}$

by assuming that the stellar mass-to-light ratio $\left(M_{\star} / L\right)$ is radially constant:

$\Sigma_{\star}(R)=\left(M_{\star} / L\right) I(R)$,

where $I(R)$ is either directly the observed surface brightness or its exponential fit. Equation (1b) corresponds to the simplest assumption according to which the stellar mass strictly follows the light. This is reasonable, especially when the surface brightness is measured in the red or infrared bands that best trace the

${ }^{1}$ For the scope of this paper we use the term "rotation curve" also in the sense of "circular velocity as a function of radius". stellar mass distribution, in view of the approximate radial constancy of galaxy colours (e.g. Kent 1986; Verheijen 1997). On the other hand, various observational facts suggest that different regions in a spiral galaxy experience different evolutionary histories that lead to different colours and mass-to-light ratios. Then, it is worth dropping the assumption of a constant $M_{\star} / L$ and investigating the effects of realistic radial variations in the $M_{\star} / L$ ratio on the mass models.

The main argument for this comes from the observed metallicity gradients: in disc galaxies (including the Milky Way) the metallicity, traced by the HII regions and by the youngest stars, decreases outward with a typical gradient of $-0.2 \operatorname{dex} h_{B}^{-1}$ in [O/H] (Garnett et al. 1997; van Zee et al. 1998; Shaver et al. 1983; Smartt \& Rolleston 1997; Gummersbach et al. 1998). The standard interpretation is that star formation and chemical enrichment proceed in the outer regions at a slower pace than in the inner regions, in an "inside-out" formation scenario (Matteucci \& François 1989; Sommer-Larsen 1991; Ryder \& Dopita 1994; Portinari \& Chiosi 1999, and references therein). This is supported by the evidence that spiral discs are systematically bluer towards their outskirts and the scalelength of the light distribution is typically shorter in red bands than in the blue (de Jong 1996c; Bell \& de Jong 2000; Jansen et al. 2000). Therefore, at a face value, stellar populations of younger age and of lower metallicity lie in the outer regions. All this points to a decline in $M_{\star} / L$ with radius (Bell \& de Jong 2001), whose extent and consequences are ripe for investigation. 
The other main player in the mass structure of galaxies is the dark halo. This can be modelled by means of a theoretical approach that stems from the favoured $\Lambda$ cold dark matter $(\Lambda \mathrm{CDM})$ cosmological scenario, very successful at accounting for the large-scale structure of the Universe. For virialized objects, this predicts well-defined "cusped" profiles (Navarro et al. 1996) that, however, have been found to be at variance with observations (e.g. Moore 1994; Salucci et al. 2003; de Blok \& Bosma 2002; Kuzio de Naray et al. 2006; de Blok 2007; Spano et al. 2008). Alternatively, we resort to an empirical approach: one can easily find a form of (cored) DM density distribution that can account for all available kinematic data (Gentile et al. 2004, 2005; Salucci et al. 2007).

The present work aims to bridge the gap between the chemophotometric studies, which support inside-out formation and radial gradients in colours and $M_{\star} / L$, and the mass modelling of spiral galaxies where $M_{\star} / L$ is traditionally assumed to be constant over the whole disc (with a few recent exceptions, see below).

Let us briefly review the state of the art of the study of the kinematics of spiral galaxies with the aid of chemo-photometric information. The average $M_{\star} / L$ ratio of a stellar disc, obtained from its global broadband colours by means of stellar population synthesis techniques, has proved to be a useful tool in dynamical studies of spiral galaxies (Ashman et al. 1993; Bell \& de Jong 2000). More recently, Kranz et al. (2003) and Kassin et al. (2006) for the first time have mass-modelled the rotation curve (RC) of a number of spiral galaxies, relaxing the assumption that $M_{\star} / L(R)=$ constant and considering the existence of (moderate) radial colour gradients. From these they derived the radial variation in $M_{\star} / L$ by means of the (allegedly universal) relationship between the $M_{\star} / L$ and colour of a mixed stellar population,

$\left\langle\log \frac{M_{\star}}{L_{K}}\right\rangle=a+b\langle(B-R)\rangle$,

found by spectro-photometric models (Bell \& de Jong 2001; Bell et al. 2003). This new treatment of the stellar disc surface density, considered just as a refinement, has seemingly brought out no major new features in the mass models of spiral galaxies. Presently, colour- $M_{\star} / L$ relations are being used in a variety of contexts where the mass profile of the stellar disc needs to be estimated (see e.g. Bakos et al. 2008; Treuthardt et al. 2009).

However, this pioneering way of accounting for variable $M_{\star} / L$ ratios is still subject to improvement. In fact, the above relation was derived from the global properties of model galaxies ${ }^{2}$; namely, the relation is supposed to hold among galaxies, relating their global colour and mass-to-light ratio. Yet, in the abovecited dynamical studies, the relation is instead applied within each object in order to estimate the local $M_{\star} / L$ from the local colour $(B-R)(R)$. Moreover, it is sometimes applied beyond the range of colours for which it was established. Some caution is therefore necessary when such colour $-M_{\star} / L$ relations are

\footnotetext{
2 The Bell \& de Jong (2001) models are detailed multi-zone models accounting for the observed gradients in colour, metallicity, and age of galactic discs; however, the colour $-M / L$ relations they present refer to their global galaxy models. Though similar relations have been shown to also hold radially, within individual discs (PST04) it is not a priori guaranteed that the same relations hold globally and locally, especially for NIR bands where the age-metallicity degeneracy is lifted. This caveat applies in particular to the (much shallower) semi-empirical relations of Bell et al. (2003), which are the result of simple one-zone models calibrated on the global photometric properties of a large sample of SDSS/2MASS galaxies.
}

applied radially within individual galaxies: in the inside-out scenario, it is likely that radial variations in $M_{\star} / L$ are far more "coherent" than the variations related to global colour from galaxy to galaxy.

In this paper we adopt a different approach to investigating the radial $M_{\star} / L$ gradients and their consequences for the mass structure of spiral galaxies. We work out, for the first time in a self-consistent way, the theoretical radial gradients in $M_{\star} / L$ of stellar discs by means of multi-zone chemo-photometric models. These follow the radial history of star formation and account for the observed metallicity and colour gradients. We obtain a typical radial profile of variable mass-to-light ratio (VML) that, convolved with an exponential light distribution, yields the underlying stellar density profile.

We derive the corresponding VML-disc contribution to the circular velocity and we mass-model the co-added rotation curves of Persic et al. (1996), that represent the average RC of a spiral of a given luminosity well (see Salucci et al. 2007, and references therein). Then, moving beyond the usual assumption of a constant mass-to-light ratio, we investigate crucial issues of the mass distribution of disc galaxies. These include the ubiquitous presence of DM in every object, the presence of a region of "inner baryon dominance", the luminosity dependence of the darkto-luminous mass fraction, and finally the inner density profile of the dark matter halo. We also discuss VML discs in the MOND scenario.

The paper is organised as follows. In Sect. 2 we derive the average typical $M_{\star} / L$ profiles from chemo-photometric models of disc galaxies. In Sect. 3 we show how they are supported by the observed colour gradients. In Sect. 4 we determine the corresponding VML-disc rotation curve; we then discuss the consequent disc/dark halo decomposition of rotation curves. Finally, in Sect. 5 we draw our conclusions.

\section{Chemo-photometric models and radial gradients in stellar mass-to-light ratio}

The standard "inside-out" scenario of disc galaxy evolution was originally developed to match a number of chemical properties in the Solar Neighbourhood and in the Milky Way (e.g. Matteucci \& François 1989; Chiappini et al. 1997, 2001; Portinari et al. 1998; Portinari \& Chiosi 1999, 2000; Boissier \& Prantzos 1999). Subsequently, it was successfully used to describe the chemical and photometric evolution of individual spiral galaxies (e.g. Mollá et al. 1996, 1997; Renda et al. 2005), as well as the whole spiral population (e.g. Boissier \& Prantzos 2000, 2001; Prantzos \& Boissier 2000; Bell \& Bower 2000; Mollá \& Diaz 2005). In this section we discuss how this scenario necessarily implies that stellar discs in spiral galaxies have radial gradients in colour and $M_{\star} / L$.

We specifically consider the multi-zone models of Portinari et al. (2004; hereinafter PST04) that compute the detailed chemical and photometric profiles of the disc and the corresponding radial $M_{\star} / L$ and colour profiles. Radial profiles were not explicitly presented in PST04 (which was focused on the global $M_{\star} / L$ of disc galaxies), so we will do it in the present paper. Let us anticipate (Sect. 2.2) the main result: when tuned to observations, chemo-photometric models imply that the disc $M_{\star} / L$ declines with radius $R$ as

$$
\frac{M_{\star}}{L}(R) \propto \exp \left\{-b\left[\left(\frac{R}{h}\right)^{s}-1\right]\right\}
$$

with $0.5 \leq s \leq 1.1$ and $0.14 \leq b \leq 0.8$, and $h$ the scalelength of the luminous profile. Any of the above cited works 
in the framework of the inside-out paradigm lead to similar $M_{\star} / L$ profiles, though this fact is rarely mentioned explicitly (one exception is Fig. 7a of Boissier \& Prantzos 1999), probably because the issue lies outside the direct scopes of chemical evolution studies.

We briefly outline the main features of the code and the relative models, but the reader uninterested in the details can directly go to Sect. 2.2 where we present $M_{\star} / L$ profiles derived from the PST04 models. In short: (1) the main observable underlying the derived mass-to-light profile is the metallicity gradient of spiral galaxies $\left(-0.2 \pm 0.1 \mathrm{dex} h_{B}^{-1}\right.$ in $[\mathrm{O} / \mathrm{H}]$, quite independent of mass and Hubble type); (2) the PST04 models correspondingly predict colour gradients that will be checked in Sect. 3 .

\subsection{Main features of the models}

Here below we briefly summarise the main features of the chemo-photometric models of PST04, but further details can be found in the original paper.

- The disc galaxy models possess a multi-zone radial structure, divided into (typically 26) concentric annuli, which allows us to follow radial gradients in star formation (SF) history. The local SF and chemical evolution history of each annulus are used to compute the radial profiles of metallicity, luminosity, and colours.

- A galactic disc is assumed to form gradually via slow accretion ("infall") of primordial gas, essentially cooling from the gaseous halo and settling into the disc. An exponentially decreasing infall rate is invoked to fit the metallicity distribution of long-lived stars in the Solar Neighbourhood (i.e. the "G-dwarf" problem; Lynden-Bell 1975; Tinsley 1980; Pagel 1997). This kind of infall is also observed in dynamical models of the formation of galactic discs (Larson 1976; Sommer-Larsen 1991; Burkert et al. 1992; Sommer-Larsen, et al. 2003).

- The present-day surface density distribution of the baryonic matter (stars+gas) is forced to an exponential radial profile. With this choice, the present-day luminosity profile also approaches an exponential, as observations indicate, and a scalelength $h$ can be confidently determined, at least out to 3-4 scalelengths (see also Boissier \& Prantzos 1999).

- The code adopts a physically motivated star formation efficiency, which increases with the local surface mass density (Dopita \& Ryder 1994; see Sect. 6 of PST04). This form of SF law is known to reproduce the radial chemical and photometric profiles of galaxies (Portinari \& Chiosi 1999; Bell \& Bower 2000).

- Once the stellar initial mass function (IMF) is fixed, the main parameters of the code are the SF efficiency $v\left(\right.$ in $\mathrm{Gyr}^{-1}$ ) and the infall timescale $\tau_{\text {inf }}$. The models are then a two-parameter family. They were calibrated to reproduce the typical average chemical properties (metallicity and metallicity gradients) of Milky Way-sized spiral galaxies, as follows. A grid of models with different infall timescales $\tau_{\text {inf }}$ is developed, and for each $\tau_{\text {inf }}$, the SF efficiency $v$ is tuned to reproduce the typical metallicity of spiral galaxies with $M_{B} \sim-21$. Specifically, they reproduce $12+\log (\mathrm{O} / \mathrm{H})=9.1 \mathrm{dex}$ at a galactocentric radius $R=1 h_{B}$ (see Sect. 6.2 of PST04). Once the absolute values of $v$ and $\tau_{\text {inf }}$ are so calibrated, we consider the radial metallicity gradient that must match the observed $-0.2 \pm 0.1 \operatorname{dex} h_{B}^{-1}$ in $[\mathrm{O} / \mathrm{H}]$ (Garnett et al. 1997; van Zee et al. 1998; Prantzos \& Boissier 2000). This is often obtained with no further tuning of the parameters. The adopted SF law in fact, with a dependence on surface density, can reproduce the observed metallicity profile. Only models with long infall timescales reach an agreement with observations by introducing an additional radial increase in $\tau_{\text {inf }}(R)$ $\left(\tau_{\text {inf }}(R) \propto R^{x}, 0.5 \leq x \leq 2\right.$, see Table 2 of PST04).

- We used six different IMFs: those of Salpeter (1955), Kroupa (1998), Kennicutt (1983), Larson (1998), and Chabrier (2001, 2002). The other IMFs are called "bottom-light" with respect to Salpeter, in that they store less mass in the lowmass end of the distribution $\left(<1 M_{\odot}\right)$ than the Salpeter IMF (extended down to $0.1 M_{\odot}$ ). These IMFs are favoured by direct estimates of the stellar mass function in the Solar Neighbourhood, in the Galactic bulge, and in globular clusters and are indirectly supported by Tully-Fisher arguments (PST04). The upper mass limits on the IMFs were, in some models, tuned to reproduce the observed gas fractions of late-type disc galaxies, but such fine tuning has very little relevance for the aims of this paper.

We thus obtained, for each of the IMFs considered, a series of models with $\left(v, \tau_{\text {inf }}(R)\right)$ calibrated to reproduce the observed metallicity of Milky Way-sized spiral galaxies, and the typical metallicity gradients (which are quite independent of mass and Hubble type).

Increasing infall timescales $\tau_{\text {inf }}$ 's "produce" objects with more extended SFHs (larger "birthrate parameters", the ratio between present-day and past average SFR) and bluer colours, that represent galaxies of later Hubble types (Kennicutt et al. 1994).

Finally, we stress that, while the absolute value of metallicity is a function of galaxy mass, the metallicity gradient is quite independent of mass and Hubble type (Garnett et al. 1997; van Zee et al. 1998; Prantzos \& Boissier 2000). Therefore, the radial trends obtained for the Milky Way-sized galaxies modelled by PST04 are actually valid in general.

Barred galaxies may be an exception, as they often present much shallower metallicity gradients (e.g. Martin \& Roy 1994), probably a result of bar-induced radial gas flows that wash out any pre-existing gradient. However, this phenomenon is probably limited to strong bars; for instance, our Milky Way hosts a bar (or two; Lopez-Corredoira et al. 2007, and references therein) yet its metallicity gradient is in line with the one we model - and has actually inspired the whole inside-out scenario since the 80's. Besides, bar-induced gas flows wash out only the present-day metallicity gradient in the gas phase, while the underlying metallicity, colour, and $M_{\star} / L$ gradients in the stellar component are much less affected, so our models should reasonably describe barred galaxies, as long as their colour gradients are similar to those of other galaxies.

Clearly, our present discussion does not apply in individual objects where no significant metallicity and colour gradients are observed (whether due to bar mixing or other reasons); however, this paper focuses on average trends and we show in Sect. 3 that the models yield reasonable predictions for the average colour gradients of disc galaxies. For the sake of completeness, we mention that radial mixing of stellar populations may also affect the chemo-photometric evolution of disc galaxies (Roškar et al. 2008; Schönrich \& Binney 2009). While very promising for understanding the detailed connections between kinematics and chemistry in Galactic stars, models including these effects are still in their infancy. Whether they represent just a refinement over the underlying inside-out scenario or will drastically change the standard interpretation of the metallicity, colour, and $M_{\star} / L$ gradients of disc galaxies remains to be explored, especially considering that such models involve many more free parameters 
than the standard ones. In this paper we necessarily limit our discussion to the dynamical consequences of the decade-long, well-established inside-out scenario.

\subsection{The radial profile of $M_{\star} / L$}

It is well known that the absolute value of $M_{\star} / L$ depends on the SF history, stellar metallicity distribution function, and underlying IMF. Global information, such as absolute values of the metallicity and colour, is very valuable to frame the galaxy properties and compare galaxies to each other - though is per se unable to constrain the mass models with a precision comparable to kinematic analysis (see Salucci et al. 2008).

However, in this paper we are not interested in differences among different objects, but focus on the internal radial profiles in $M_{\star} / L$ and their dynamical consequences compared to the simple constant $M_{\star} / L$ assumption. Therefore, we shall adopt a suitable scaling that cancels out object-to-object offsets and highlights the radial $M_{\star} / L$ variation within the discs.

We define the $I$ band scalelength $h_{I}$ by fitting the model $I$ band luminosity profile with the exponential law of Eq. (1a) in the range $0.5-3 h_{I}$. Introducing the notation $M_{\star} / L_{\lambda}(R) \equiv$ $\Upsilon_{\lambda, *}(R)$, we take the value of the $M_{\star} / L$ at one scalelength, $\Upsilon_{\lambda, 1}=M_{\star} / L_{\lambda}\left(h_{I}\right)$ as the zero-point normalization of the $M_{\star} / L$ and we define the normalized $M_{\star} / L$ radial profile as

$$
\frac{\Upsilon_{\lambda, \star}(R)}{\Upsilon_{\lambda, 1}}=\frac{\frac{M_{\star}}{L_{\lambda}}(R)}{\frac{M_{\star}}{L_{\lambda}}\left(h_{I}\right)}
$$

The normalization eliminates the zero-point offsets related to the specific IMF and star formation history of each model. The normalized $M_{\star} / L$ profiles of the models show very similar behaviour, with representative examples shown in Fig. 1. The profiles range from "shallow" (close to linear out to $\sim 3 h$ and shallower farther out; top panel) to "concave" (bottom panel).

In any case, $M_{\star} / L$ decreases with radius in all models, simply as an effect of the age and metallicity gradients of the stellar populations that evolve in an inside-out scenario. Quantitatively, $M_{\star} / L_{I}$ decreases by about a factor of 2 between 1 and 3-4 scalelengths, with a rise towards the centre for the concave cases. Though the radial gradients in $M_{\star} / L$ are stronger in bluer bands, they are also non-negligible in the $K$ band: $M_{\star} / L_{K}$ decreases by about $40 \%$ between 1 and $4 h_{I}$. As a result, the stellar surface density profile is generally more concentrated than the brightness profile, even in the NIR (see also Kranz et al. 2003).

To proceed, we first consider that the $I$ band offers a number of observational and theoretical advantages (see PST04), so that we choose it as the reference band - as in the kinematic analysis of Sect. 4.1. We find that the normalized $M_{\star} / L_{I}$ profiles are all approximated well by

$\frac{\Upsilon_{I, *}(R)}{\Upsilon_{I, 1}}=\exp \left\{-a(s)\left[\left(\frac{R}{h_{I}}\right)^{s}-1\right]\right\}$

with

$a(s)=\frac{5}{4}(1.3-s)^{3}+0.13$.

In detail, shallow $M_{\star} / L_{I}$ profiles have $s \simeq 1(0.9-1.1)$, i.e. close to exponential, while concave profiles are well-fitted by $s=0.5-0.7$; e.g., the example concave profile in the bottom panel of Fig. 1 corresponds to $s=0.5$. The standard constant mass-to-light assumption is recovered for $s=0$.
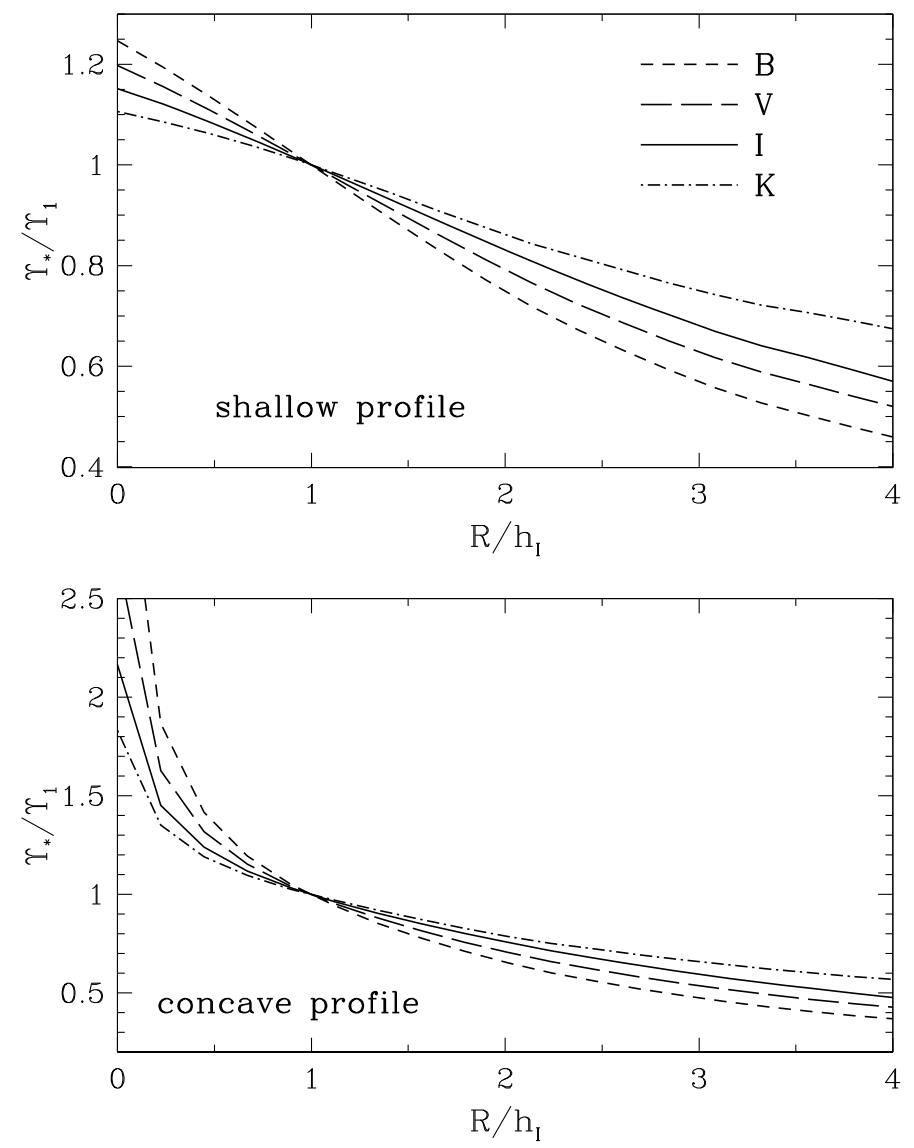

Fig. 1. $M_{\star} / L$ profiles in different bands predicted by our chemophotometric models, scaled to the respective values at $R=h_{I}$ (Eq. (2)). An example of $M_{\star} / L$ with shallow profile (model Kenn-H of PST04, top panel) and one with concave profile (model Chab-F, bottom panel) are shown.

The analytical fit in Eq. (3) is accurate to $2-3 \%$ at $R \geq 0.5 h$ and to about $10 \%$ in the very central regions. For the typical "average" spiral galaxy the actual value of $s$ will lie within the (reasonably narrow) range of values discussed above. In passing, let us point out that it is up to future investigations to test whether Eq. (3) can be also representative of individual objects, and determine the corresponding values of $s$.

\section{Colour gradients}

It is well known that spectro-photometric models of spiral galaxies predict tight relations between the $M_{\star} / L$ and the colours of (simple and composite) stellar populations (Bell \& de Jong 2001; PST04). Henceforth, the observed colour gradients in spiral galaxies can be considered the empirical counterpart of the $M_{\star} / L$ gradients. An obvious test for our model $M_{\star} / L$ profiles is then to compare the corresponding colour profiles, predicted to get bluer outwards, to observations. To this purpose we consider the multi-band sample of 86 disc galaxies of de Jong \& van der Kruit (1994). It is worth noting that the overall colour of a spiral galaxy tends to be redder for earlier morphological types (e.g. Roberts \& Haynes 1994) because of their different average disc SF histories, rather than to systematic variations in the bulge-to-disc ratios (Kennicutt et al. 1994; de Jong 1996b,c). To circumvent this intrinsic variance in the global colour of different galaxies, and highlight the radial trends within each of them, 


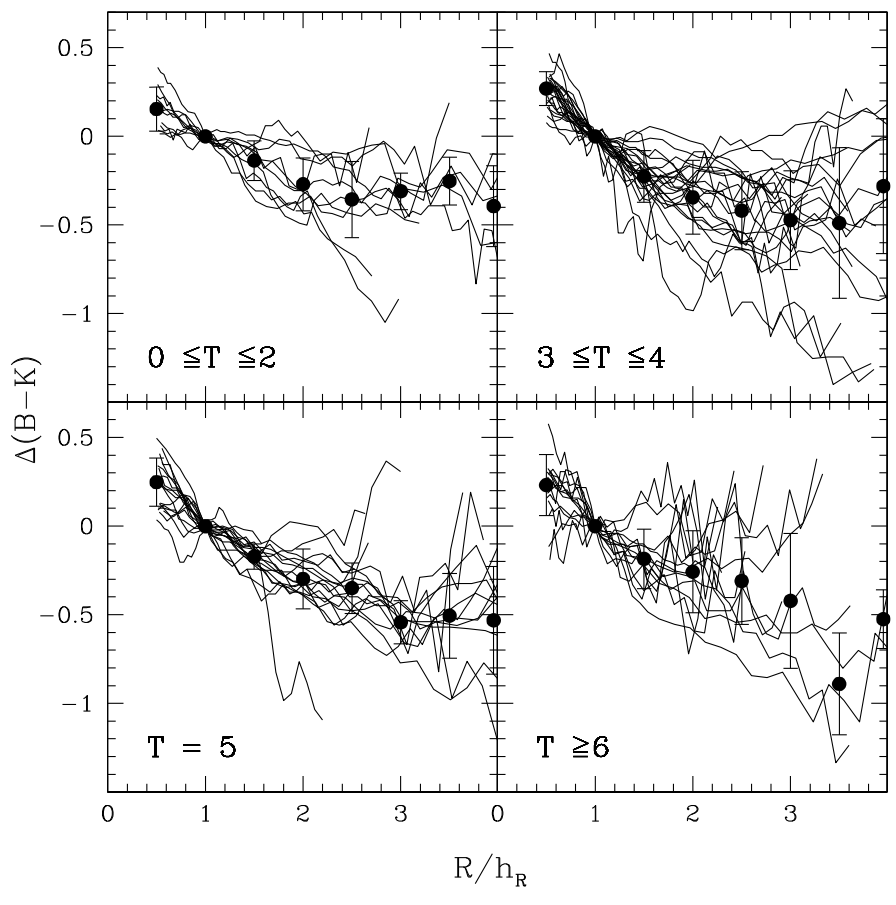

Fig. 2. Colour difference profiles for the sample of disc galaxies of de Jong \& van der Kruit (1994). $T$ codes the galaxy morphological type: $T=0-2(\mathrm{Sa}-\mathrm{Sab}), T=3-4$ (Sb-Sbc), $T=5(\mathrm{Sc}), T=6-10(\mathrm{Scd}$ and later types). Dots with error bars represent the average $\Delta$ (colour) and its $1 \sigma$ dispersion for the co-added profiles.

we normalize the colour data by taking the colour measured at 1 scalelength $h$ as the zero-point and by shifting all the values as

$\Delta($ colour $)=\operatorname{colour}(R)-\operatorname{colour}(h)$.

This shift is consistent with the $M_{\star} / L$ scaling of Eq. (2) that we apply to the models.

We show the resulting "colour difference profiles" in Fig. 2, which is obtained from Fig. 2 of de Jong (1996c) after the zeropoint shift by expressing the radial coordinate in disc scalelengths (taken from de Jong 1996a). For the sake of example we show the run of $B-K$ colour versus radius measured in $R$-band scalelengths, but similar trends hold in other bands (see also Fig. 3). We ignore the data of the innermost half scalelength, which are heavily affected by the bulge component. Figure 2 clearly highlights the existence of negative radial colour gradients, out to at least 3 scalelengths. For different morphological types the colour gradients are quite similar in the range $0.5 \leq R / h \leq 3$, with Scd and later type galaxies showing a larger scatter. Beyond $3 h$ the colour profiles become shallower (and noisy).

In spite of the considerable scatter (and of uncertainties in sky background subtraction at the outermost radii), there is no question that the colours on average become bluer outwards; $B-K$, for example, decreases by about 0.25 mag per disc scalelength. From this, we can claim that colour gradients emerge as a systematic, constitutive property of galactic discs.

Then, in Fig. 3 we overlay the colour profiles of all morphological types $0 \leq T \leq 6$ (neglecting later, more irregular types) and we compare their average values with the predictions of our chemo-photometric models. In detail, we plot various colour profiles in different bands and compare the data with the model predictions obtained by assuming a variety of IMFs. Each panel shows, for a given set of IMF models, two examples of model colour gradients, the shallower/steeper one corresponding to the shallow and concave $M_{\star} / L$ profiles in Sect. 2.2.

For all the adopted IMFs, a good agreement results between the colour profiles predicted by the models for an "average" spiral and the actual observations. Therefore, in the inside-out formation scenario of stellar discs, colour gradients emerge naturally and are remarkably similar to the observed ones.

Notice that we neglect the innermost $0.5 \mathrm{~h}$ region, where the comparison would be inappropriate, as data is affected by a bulge component, not included in our models. It is not our aim to study bulge-dominated discs or the bulge-disc interface, and since the innermost $R<0.5 h$ region encloses only $10-15 \%$ of the disc mass, the lack of a more exact treatment does not significantly bias our investigation on VML-discs.

In conclusion, the observational properties of stellar discs indicate that their $M_{\star} / L$ is likely a function of radius and that chemo-photometric models predict the average radial colour and $M_{\star} / L$ profiles of spiral galaxies reasonably well.

\subsection{Dust effects}

In our models and in the comparison with the observed colour profiles, we have neglected the effects of dust. This is to a first approximation justified, since the observational sample consists of face-on galaxies for which dust has been found to play a minor role in the colour gradients (de Jong 1996c). For an extended sample of low inclination galaxies, Bell \& de Jong (2000) similarly conclude that colour profiles are driven by gradients in the age and metallicity of the stellar populations; dust induces a much smaller additional gradient. Besides, again to first approximation, dust does not alter the colour $-M_{\star} / L$ relation (the reddening-extinction dust vector running almost parallel to the relation, see Bell \& de Jong 2001). Therefore the $M_{\star} / L$ profiles corresponding to the observed colour profiles are also expected to be mainly driven by stellar population gradients rather than by dust effects.

On the theoretical side, the chemo-photometric model of Boissier \& Prantzos (1999) for the Milky Way also predicts that dust slightly enhances, but does not create colour gradients. As their models are quite similar to those of PST04, we take the results from their Fig. 7 to illustrate the point. In Fig. 4 we show the $M_{\star} / L_{V}$ and the colour profiles of the Milky Way model of Boissier \& Prantzos (1999): dust-free predictions and (face-on) extinction. The left panels reproduce their Fig. 7, with the radial coordinate changed from $R$ to $R / h_{R}$, where $h_{R} \sim 3 \mathrm{kpc}$ (estimated from the surface brightness and colour profiles in their Fig. 7). The $R$-band scalelength should be quite close to the $I$-band scalelength, which we refer to in our discussion of the $M_{\star} / L$ gradients and rotation curves. The right panels show the same $M_{\star} / L_{V}$ and colour profiles, but with the relative scalings adopted in Sects. 2.2 and 3. Clearly the absolute values of colours and $M_{\star} / L$ are affected by dust (left panels), but in relative terms, the effect on the colour gradients is small with respect to the amplitude of the gradient. The strongest effect is on optical-NIR colours like $B-K$, as noticed by de Jong (1996c). Comparison to Fig. 3 shows that the effect of dust on the colour gradients is much less than the scatter among individual galaxies, and it does not significantly change the discussion in the previous section. As to $M_{\star} / L_{V}$, dust enhances the gradient but remains a second-order effect with respect to the main trend set by the inside-out scenario; and extinction affects the $I$-band (relevant for the remainder of our paper) much less than the $V$-band.

In summary, dust certainly plays a non-negligible role when discussing the absolute values of colours, $M_{\star} / L$ ratios, and age 

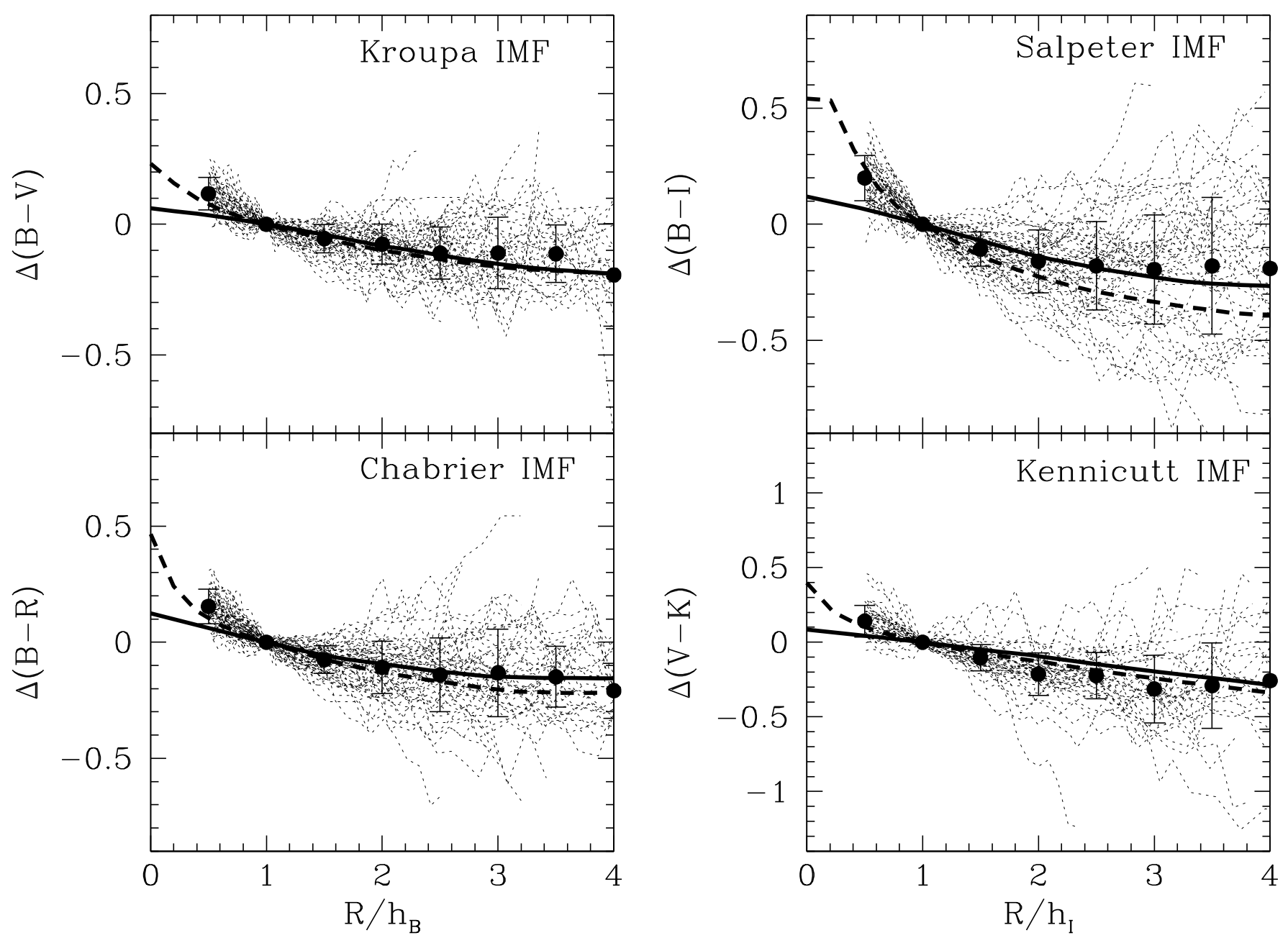

Fig. 3. Radial colour difference profiles in different bands, computed relative to the colour at $R=h$ (Eq. (4)). Thin dotted lines: data from de Jong $\&$ van der Kruit (1994) for $0 \leq T \leq 6$; dots with error bars: corresponding average $\Delta$ (colour) and $1 \sigma$ dispersion; thick solid and dashed lines: chemo-photometric models from PST04 (specifically, the models plotted are Krou-C,E; Salp-A,E; Chab-A,F; Kenn-H,J).

of the underlying stellar populations, but its role is secondary in terms of the gradients discussed in this paper. Dust effects certainly deserve further attention, especially for inclined galaxies, but they will not change our main point. Radial $M_{\star} / L$ gradients due to stellar populations must exist according to the inside-out scenario, and they significantly change the disc rotation curve with respect to the classic Freeman one (Sect. 4). From Fig. 4, we expect that dust at most enhances the trends we discuss.

\section{Variable mass-to-light rotation curve}

To establish the actual importance of the radial variations of the stellar $M_{\star} / L$, a complete chemo-photometric investigation of a large sample of individual objects would be needed, which is beyond our present scope. Here we aim to investigate the effects of the average stellar $M_{\star} / L$ gradient of spiral galaxies (Eq. (3)), in connection with their average rotation curve (RC), defined as the co-added curve of a suitably large sample of RC's of objects of fixed luminosity.

From now on, we specifically consider the $I$ band, which is the reference band for the set of rotation curves studied in Sect. 4.1 and drop the subscript $I$ for simplicity. From Eq. (3) for the $M_{\star} / L$ profile we can build the average luminous component of the gravitational potential of spiral galaxies. We consider values of $s=0.5,0.7$, and 1.0 (Fig. 5), which cover the range of profiles obtained from the models.

We assume that the Freeman profile (Eq. (1a)) describes the light distribution. The (small) deviations we observe in the surface brightness of some spirals are important for individual mass modelling but irrelevant for the present purposes. As a consequence of the radial gradients in $M_{\star} / L$, the stellar surface density $\Sigma_{\star}(R)$ is somewhat more concentrated than the surface luminosity profile. For an exponential disc (Eq. (1a)), from Eq. (3) we get

$\Sigma_{\star}(R)=\Sigma_{1} \exp \left\{-a(s)\left[\left(\frac{R}{h}\right)^{s}-1\right]-\left[\left(\frac{R}{h}\right)-1\right]\right\}$

where $\Sigma_{1}=\Sigma_{\star}(h)=\Upsilon_{1} I_{0} / e$ is the surface density at 1 scalelength. For a given $\Sigma_{1}$, the surface density has a central value $\Sigma_{\star}(0)=\Sigma_{1} e^{a+1}$, higher than that of the $M_{\star} / L=$ constant case by a factor $e^{a}(\simeq 1.2-2$ for $s$ in the range $0.5-1.1)$, and decreases more rapidly outward than in the standard mass-follows-light assumption. In the case of $s=1$, the surface density still has an exponential profile, but with scalelength $h /(1+a)$, i.e. about $20 \%$ shorter than that of the surface brightness profile. In this case we note that the main VML feature, a mass scalelength slightly less than the light scalelength, may have been so far masked by the intrinsic difficulties in deriving the surface brightness scalelength $h$ well within the $20 \%$ precision level. 
L. Portinari and P. Salucci: Mass distribution of spiral galaxies: considering the radial profiles in $M_{\star} / L$
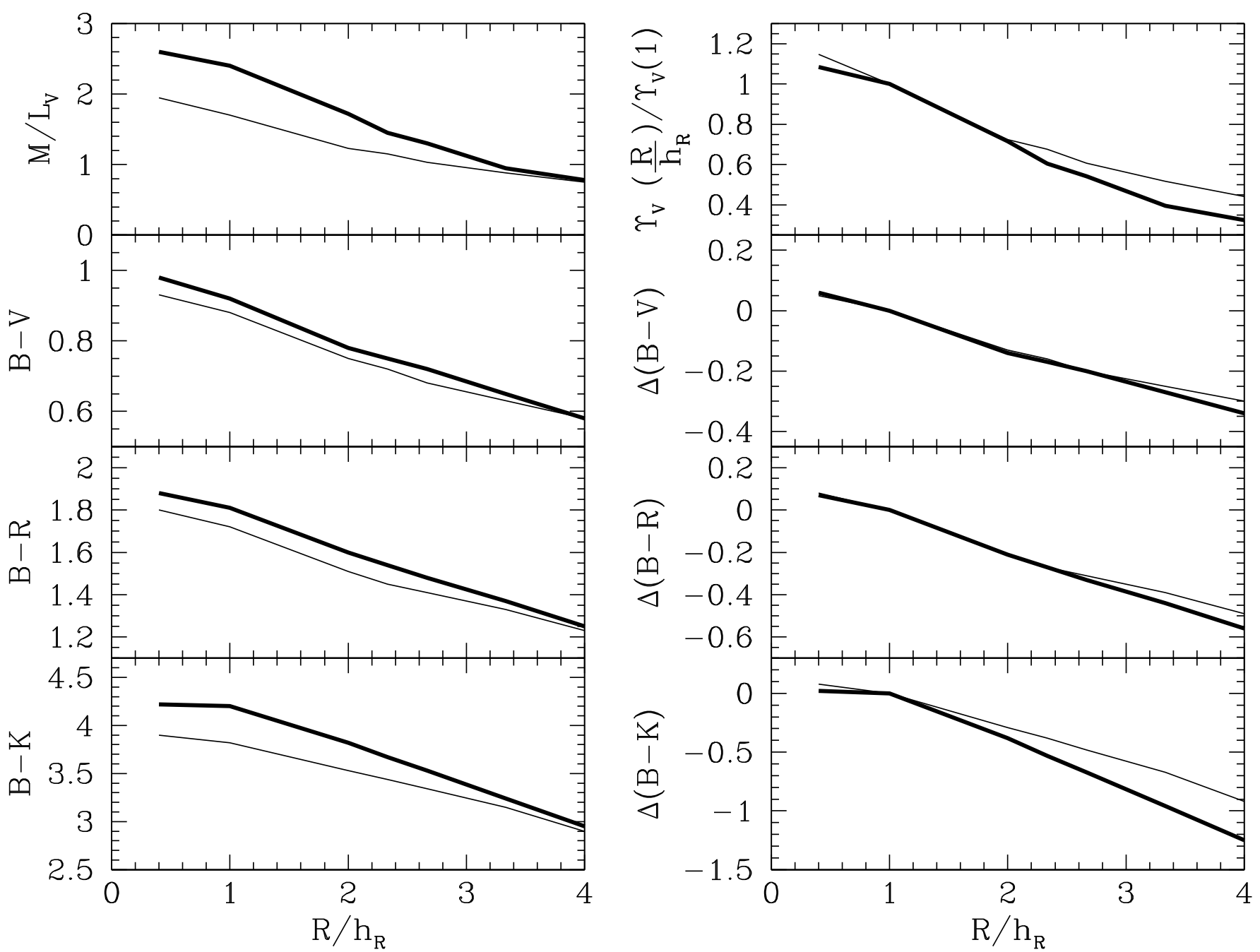

Fig. 4. Radial $M_{\star} / L_{V}$ and colour profiles from the Milky Way model of Boissier \& Prantzos (1999); thin lines: dust-free predictions; thick lines: including extinction. The right panels show the relative $M_{\star} / L_{V}$ and colour profiles, scaled as discussed in Sects. 2.2 and 3.

In Fig. 5 we show three analytical $M_{\star} / L$ profiles with $s=$ $0.5,0.7$, and 1.0, and in Fig. 6 we show the corresponding cumulative disc masses compared to the constant $M_{\star} / L$ (CML) case. The radial mass build-up is obviously more rapid and saturates more quickly in the VML case, with up to $70 \%$ of the total mass contained within about $2 h$ and up to $90 \%$ of the mass within $R_{\text {opt }}=3.2 \mathrm{~h}$.

By solving the appropriate Poisson equation we compute numerically the self-equilibrium circular velocity of thin discs with a VML surface density as given in Eq. (5). In Fig. 7 we show the resulting rotation curves for the three representative VML discs of Figs. 5 and 6, compared to the standard CML rotation curve. The VML RC rises more steeply and peaks at smaller radii (1.5$1.9 h)$ than the Freeman CML rotation curve $(2.15 h)$, and it falls off also more rapidly after the peak. The VML RCs approach the corresponding Keplerian fall-off (defined as the region where the logarithmic slope of the RC is close to -0.5) already at $R=3-3.5 h$, rather than at $R>4 h$ as it occurs for the CML disc; for $R \simeq 5 h$ the VML RCs finally overlap with the CML RC.

It is useful to provide, for the general VML RCs, an analytical fit out to $3.5 \mathrm{~h}$ :

$V_{\mathrm{VML}}^{2}(R)=V_{\mathrm{VML}}^{2}(h) n(s) \frac{\xi^{0.9+0.4 s}}{\left(\xi^{2}+(1.35+0.5 s)^{2}\right)^{1+0.3 s}}$ with $\xi=R / h$ and $n(s)=\left(1+(1.35+0.5 s)^{2}\right)^{1+0.3 s}$ a normalization factor. The fit is precise to better than $2 \%$ between $0.3 \leq \xi \leq 3.5$, and better than $10 \%$ down to $\xi \geq 0.15$. At larger radii, the VML RCs are described well by a Keplerian fall-off. The relation between the total disc mass and the value of the circular velocity at $R_{\mathrm{m}}=2.15 \mathrm{~h}$, the radius where the CML disc $\mathrm{RC}$ has its maximum, reads as

$M_{\mathrm{D}}=\eta G^{-1} V^{2}\left(R_{\mathrm{m}}\right) R_{\mathrm{m}}$

with $\eta=(0.98,1,1.03)$ for the cases of $s=(0.5,0.7,1)$, and $\eta=1.2$ for the Freeman CML disc.

\subsection{The dark-luminous matter interplay}

The stellar surface density $\Sigma_{\star}(R)$ in spiral galaxies is then only exponential to a first approximation; more in detail, it is an exponential modulated by the $M_{\star} / L$ gradient, as given by Eq. (5). $V_{\mathrm{VML}}(R)$ is somewhat different from the standard CML rotation curve. The difference is moderate for the "average" spiral as defined in this work, but in individual objects, it may not be so. From the wide range of colour profiles observed in individual galaxies (e.g. Figs. 2 and 3), we expect cases in which the VML effect is more relevant than the average case considered here, while it will be negligible in other cases. 


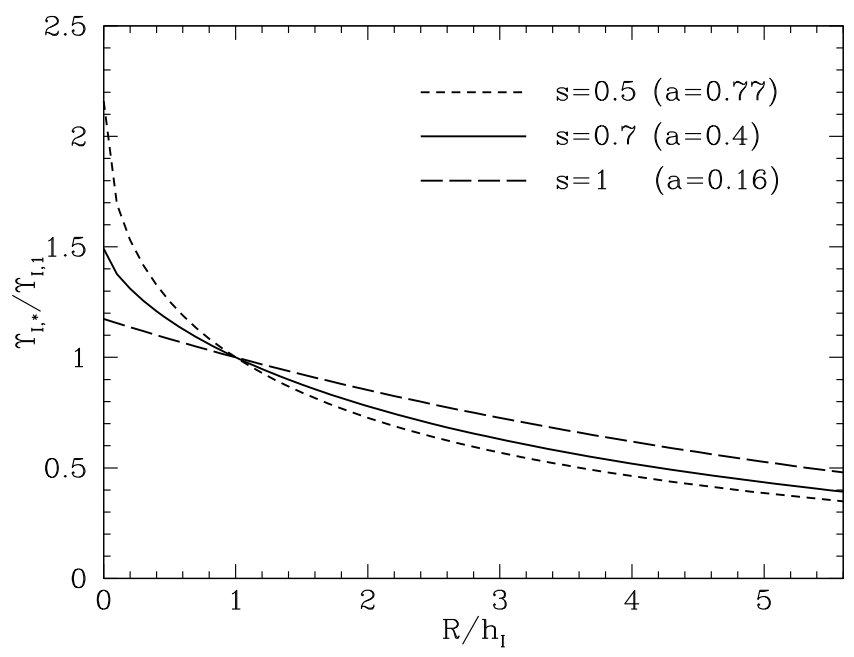

Fig. 5. Three representative examples of analytical $M_{\star} / L_{I}$ profiles derived from the chemo-photometric models (Eq. (3)).

Here, we take the function in Eq. (6) as the typical average contribution to the circular velocity of VML stellar discs. We combine it with a simple halo distribution, to mass-model the typical RCs of disc galaxies. Our main aim here is to compare the corresponding disc/halo decomposition to the results of the standard CML assumption; namely, we perform a differential analysis, rather than making absolute claims on disc fractions, halo profiles, etc.

We consider the co-added rotation curves of Persic et al. (1996, hereinafter PSS96). These are obtained by binning a very large number of individual high surface-brightness, late type, bulge-free RCs (see also Salucci et al. 2007, for references of independent work on this issue). One advantage of testing a template of co-added RCs with a template VML disc-velocity contribution is that this allows weak systematic VML kinematic features to be emphasized also, which in individual RCs would be below the noise level created by modelling uncertainties and/or observational errors. On the other hand, relevant VML features occurring in a fraction of objects with strong colour gradients can only be studied in individual RCs. Ultimately, it is in the latter that the proof for the existence of VML discs must be found.

We assume the simplest possible dark-halo velocity contribution (see PSS96):

$V_{\text {halo }}^{2}=V_{\text {opt }}^{2}(1-\beta)\left(1+\alpha^{2}\right) \frac{x^{2}}{\left(x^{2}+\alpha^{2}\right)} \quad x=\frac{R}{R_{\text {opt }}}=\frac{\xi}{3.2}$

with $R_{\mathrm{opt}}=3.2 h_{I}, V_{\mathrm{opt}}$ the observed circular velocity at $R_{\mathrm{opt}}$ and $(1-\beta)^{1 / 2}$ the fractional contribution to $V_{\text {opt }}$ of the halo component. Correspondingly, the disc contribution (whether VML or CML) is normalized so that $V_{\text {disc }}^{2}\left(R_{\text {opt }}\right)=\beta V_{\text {opt }}^{2}$.

Although simple, the function in Eq. (7) has several merits. First, inside $4 h$, it represents the NFW velocity profile for $\alpha \leq 0.2$. Moreover, for appropriate choices of the parameter $\alpha$, it also mimics the pseudo-isothermal and the Burkert (1995) halo velocity profiles. Then, it is worth recalling that, for any value of its structural parameters $(\alpha$ and $\beta$ ), the slope of our halo velocity law (and of the profiles quoted above) is very different from that of the disc contribution. At any radius we have

$\left|\frac{\mathrm{d} \log V_{\text {halo }}(R ; \alpha, \beta)}{\mathrm{d} \log R}-\frac{\mathrm{d} \log V_{\text {disc }}(R ; \beta)}{\mathrm{d} \log R}\right|>\Delta\left|\frac{\mathrm{d} \log V(R)}{\mathrm{d} \log R}\right|$

where the righthand side is the uncertainty with which we measure the RC slope. This ensures that, in the case a) of

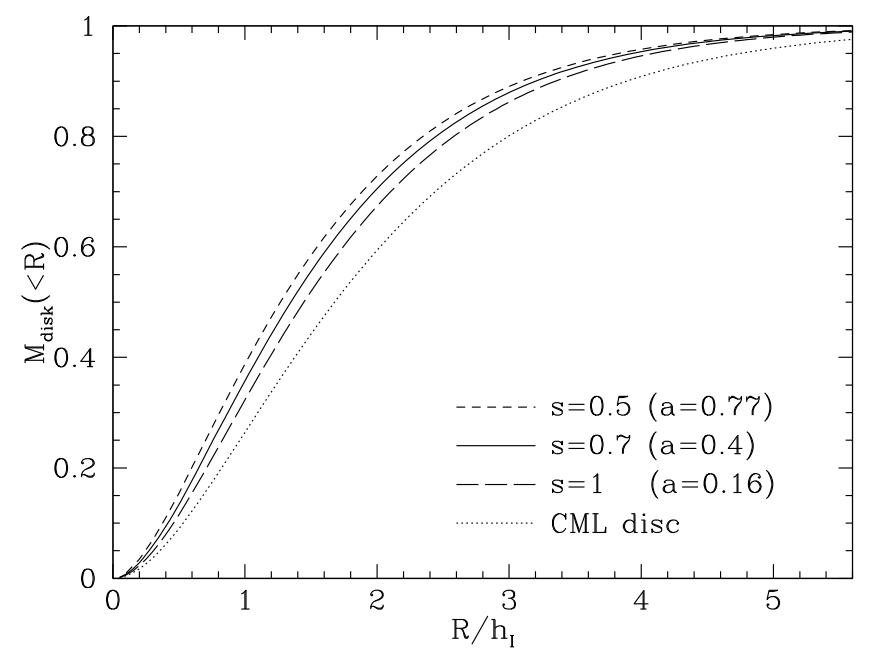

Fig. 6. Cumulative disc masses corresponding to the $M_{\star} / L_{I}$ profiles in Fig. 5, compared to the constant mass-to-light (CML) assumption.

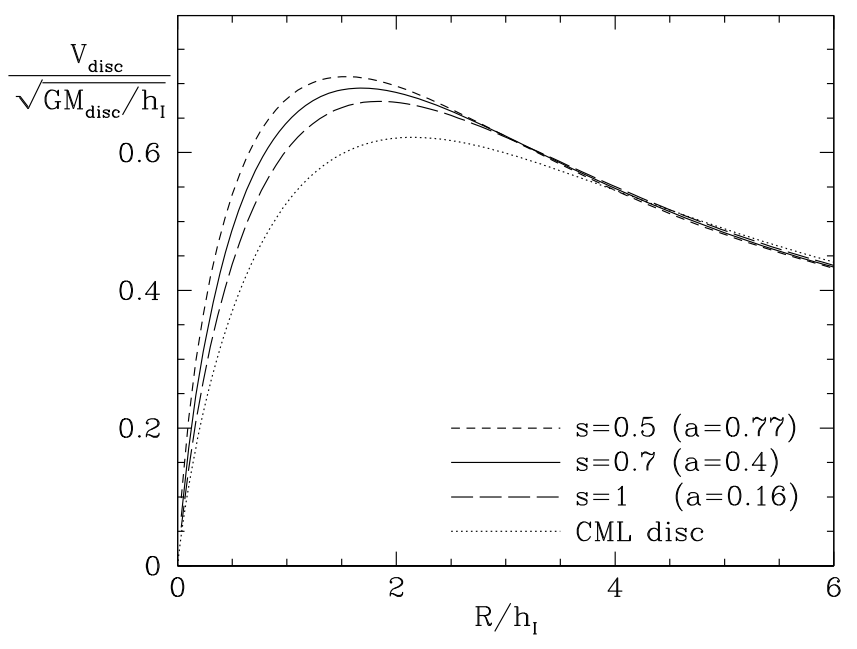

Fig. 7. Three representative examples of new VML disc rotation curves (solid and dashed lines) compared to a constant $M_{\star} / L(\mathrm{CML})$ Freeman disc (dotted line). All velocities are normalized to the respective total disc mass and $I$ band photometric scalelength.

high-quality rotation curves $(\Delta \mid \operatorname{dlog} V(R) / \mathrm{d} \log R) \mid<0.04)$; and b) we know the actual distribution of the luminous matter, the mass model we obtain by $\chi^{2}$ fitting the $\mathrm{RC}$ is essentially unique. Values of $\alpha$ and disc mass significantly different from the best-fit ones, imply mass models that significantly fail to fit the observed rotation curve. In the mass modelling we neglect the gas contribution to the RC since it is always negligible out to $\sim 3 h-$ the region where the differences between VML and CML RCs are maximal.

We fit the co-added RCs $V_{\text {coadd }}(x)$ with our 2-free parameters velocity model: $V_{\text {model }}^{2}=V_{\text {halo }}^{2}(x ; \alpha, \beta)+V_{\mathrm{VML}}^{2}(x ; \beta)$, with $V_{\mathrm{VML}}$ given by Eq. (6). Let us recall that the co-added RCs we adopt here have been modelled in PSS96 with the same halo velocity profile of Eq. (7) plus a CML exponential thin disc. The mass models obtained there (shown in Fig. 6 and in Eqs. (11)a,b of PSS96) will serve as a gauge for the models we obtain with the present investigation.

We perform $\chi^{2}$ analysis: the fitting parameters $(\alpha$ and $\beta$ ) are optimized with a Leverberg-Marquardt algorithm (Press et al. 1992). The increase in the rms scatter in the velocity data beyond $R_{\text {opt }}$ comes from the fact that, while out to $R_{\text {opt }}$ the template 
Table 1. Parameters $\alpha$ and $\beta$ and inner baryon dominance radius of the disc/halo decompositions in Fig. 8, for CML and VML disc RCs.

\begin{tabular}{lcccccc}
\hline \hline & \multicolumn{3}{c}{$\left\langle\mathrm{M}_{I}\right\rangle=-19.4$} & \multicolumn{3}{c}{$\left\langle\mathrm{M}_{I}\right\rangle=-20.9$} \\
& $\alpha$ & $\beta$ & $\frac{R_{\mathrm{IBD}}}{R_{\mathrm{opt}}}$ & $\alpha$ & $\beta$ & $\frac{R_{\mathrm{IBD}}}{R_{\mathrm{opt}}}$ \\
\hline VML (s=1.0) & 0.87 & 0.17 & 0.32 & 0.92 & 0.39 & 0.76 \\
VML (s=0.7) & 0.80 & 0.13 & 0.25 & 0.75 & 0.30 & 0.55 \\
VML (s=0.5) & 0.75 & 0.10 & 0.20 & 0.61 & 0.21 & 0.36 \\
\hline CML & 0.94 & 0.24 & 0.42 & 1.22 & 0.53 & 1.08 \\
\hline \hline \multicolumn{4}{c}{$\left\langle\mathrm{M}_{I}\right\rangle=-22.4$} & \multicolumn{4}{c}{$\left\langle\mathrm{M}_{I}\right\rangle=-23.1$} \\
& $\alpha$ & $\beta$ & $\frac{R_{\mathrm{IBD}}}{R_{\text {opt }}}$ & $\alpha$ & $\beta$ & $\frac{R_{\mathrm{IBD}}}{R_{\mathrm{opt}}}$ \\
\hline VML (s=1.0) & 1.28 & 0.64 & 1.34 & 1.29 & 0.69 & 1.53 \\
VML (s=0.7) & 0.94 & 0.52 & 1.05 & 0.93 & 0.57 & 1.18 \\
VML (s=0.5) & 0.75 & 0.42 & 0.81 & 0.75 & 0.46 & 0.91 \\
\hline CML & 1.63 & 0.80 & 1.93 & 1.71 & 1.85 & $>2$ \\
\hline
\end{tabular}

velocities come from the co-addition of 616 individual curves, at outer radii they are obtained in a different, non-trivial way (see Fig. 3 and Sect. 4 of PSS96). Then, the related rms scatter is larger and consequently the velocity data for $R>R_{\mathrm{opt}}$ do not contribute much toward setting the best-fit velocity model.

The resulting best-fit solutions for 3 different VML cases, which progressively depart from the CML one, are given in Table 1 and in Fig. 8, compared to the CML solution. As a result of the true universality of the RCs of spiral galaxies, the rms errors of the co-added curves are very small: $\Delta V / V<0.04$ (for $R<3 h$ ). Within the 2-component halo + disc model, this guarantees that the $1 \sigma$ fitting uncertainties on the parameters of any velocity model that fits the data well will be also small. In fact, we find that the $1 \sigma$ contour fitting uncertainty on $\alpha$ and $\beta$ is between $15 \%$ and $30 \%$, not surprisingly similar to the corresponding uncertainties of the CML + halo mass model in PSS96.

First of all we notice that, when also considering the fitting uncertainties, the VML stellar disc contribution to the total RC is different from the CML disc contribution. At its peak, the amplitude is lower by $\sim 20 \%$. Noticeably, the VML discs (in combination with the PSS96 DM halo profiles) reproduce the kinematic data in an excellent way: the resulting $\chi^{2}$ values are not higher than those of the CML models in PSS96; that is to say, the VML hypothesis is by no means excluded by the data. With respect to the standard CML decomposition, the overall mass distribution of spiral galaxies inferred in the VML scenario is not drastically modified, but some major differences are present. These emerge beyond the uncertainties on the structural parameters, but rely of course of the fundamental assumption that Eq. (6) holds for most spiral galaxies.

The VML disc mass distribution has a higher percentage of its total mass residing in the inner regions (i.e. $70 \%$ within $R<$ $2 h_{I}$, Fig. 6). The disc normalization, constrained by kinematic data, is lower and the inferred total disc mass and $M_{\star} / L$ ratio are about 1.5 times lower than in the CML case.

In low-luminosity spiral galaxies (Fig. 8a) dark matter already dominates the mass distribution at small radii, so that fitting with VML discs does not influence the inferred halo properties. At higher luminosity, however, the role of the disc becomes more prominent. Since the disc normalization is lower and the VML RC, after its peak at $R \sim 1.7 h$, falls off more rapidly than the CML RC, in the region $R>0.5 R_{\text {opt }}$ the dark halo velocity contribution must rise more steeply, to match the (typically still rising) rotation curve.

For galaxies of $M_{I}=-21$ and brighter, the PSS96 mass models showed a region of inner baryon dominance (IBD). Defining the corresponding radius $R_{\mathrm{IBD}}$ as the region where the baryons contribute more than $50 \%$ of the observed circular velocity, the CML mass models yield $R_{\mathrm{IBD}}$ as wide as $R_{\text {opt }}$ or more (see Table 1). Mass models based on VML discs + PSS96 dark haloes also show an IBD region, but its size is smaller: $R_{\mathrm{IBD}}$ extends beyond $R_{\text {opt }}$ only in objects of highest luminosity (Figs. 8b, c, d). In the luminosity bin of Milky Way-like galaxies $\left(M_{I}=-22.3\right.$, Flynn et al. 2006), the luminous and DM contribution to the rotation curve at $R_{\mathrm{opt}}$ are comparable, rather than still discdominated, as is the case for the CML mass models.

We find, as in PSS96, that the parameters $\alpha, \beta$ and galaxy luminosity are closely related, so we expect that scaling laws relating disc and halo masses, luminosity, disc scalelengths, and other galaxy global quantities, still hold in the VML scenario. However, we do not attempt to give explicit fitting formulae (similar to those in PSS96) because the differences we find with the PSS96 results are moderate. Moreover, the VML scenario needs yet to be confirmed by combined kinematic/chemophotometric analysis of individual objects - where scatter is large (Figs. 2 and 3).

For galaxies in the highest luminosity bin, there may be some weak feature in the co-added RC and in individual RCs that may be traced back to a VML disc. At $1-2 h_{I}$, the CML +halo rotation curve slightly overpredicts $V(R)$ at a $1 \sigma$ level in 2-3 velocity data. The VML+halo model instead matches all the data (see Fig. 6 of PSS96 and Figs. 8c, d). Moreover, at $R \sim 3 h$, VML+halo model RCs tend to remain flat, rather than decline like the CML+halo curves (Figs. 8c, d). This is not strongly excluded by the co-added curves (due to the larger error bars beyond $R_{\mathrm{opt}}$ ) and could be in line with the RC profile of some fast rotators (Spekkens \& Giovanelli 2006).

The investigation of the core vs. cusp issue in spiral galaxies requires the mass modelling of individual RCs; however it is worth discussing whether, with VML discs, the NFW haloes may become compatible with the observed kinematics. Mass modelling of co-added RCs with a CML disc + the "neutral" halo of Eq. (7) yields a core in the DM density profile; such core is similar to that found by the (much more decisive) analysis of the kinematics of selected individual objects. By replacing the CML disc with a VML one, we still find almost the same density core; although its size is about $30 \%$ smaller, it is still large enough to exclude the NFW profile beyond the uncertainties in the fitting method. Thus, at face value, VML discs cannot reconcile $N$-body predictions and observations.

Furthermore, we can resort to the ingenious proof of incompatibility with the observed kinematics given by Salucci (2001), to show that VML discs are more of a problem than a solution for NFW halos. The argument involves comparing the slope of the observed RC's with the values predicted by the NFW profile. We here apply this line of reasoning at the radius where the disc RC has its maximum, rather than at $R_{\mathrm{opt}}$ as in Salucci (2001). The following chain of facts and evidence leads to the inevitable conclusion that the DM distribution, at least in low luminosity spiral galaxies, is shallower than that predicted by a NFW halo:

i) At the radius in which the disc contribution peaks $(2.2 h$ for CML discs) and the disc RC has a flat slope, we have

$$
\left.\frac{\mathrm{d} \log V_{h}}{\mathrm{~d} \log R}\right|_{2.2 h}=\left.\left(1-\beta_{2.2}\right)^{-1} \frac{\mathrm{d} \log V}{\operatorname{dlog} R}\right|_{2.2 h}
$$

with $\beta_{2.2}=\left.\left(V_{d}^{2} / V^{2}\right)\right|_{2.2 h}$ the fractional disc contribution to $V^{2}$ at $2.2 \mathrm{~h}$.

ii) A generous estimate is $\beta_{2.2} \geq 0.15$.

iii) For NFW haloes it is $\operatorname{dlog} V_{\mathrm{NFW}} / \mathrm{d} \log R<0.2$ for $R>1.5 h$. 


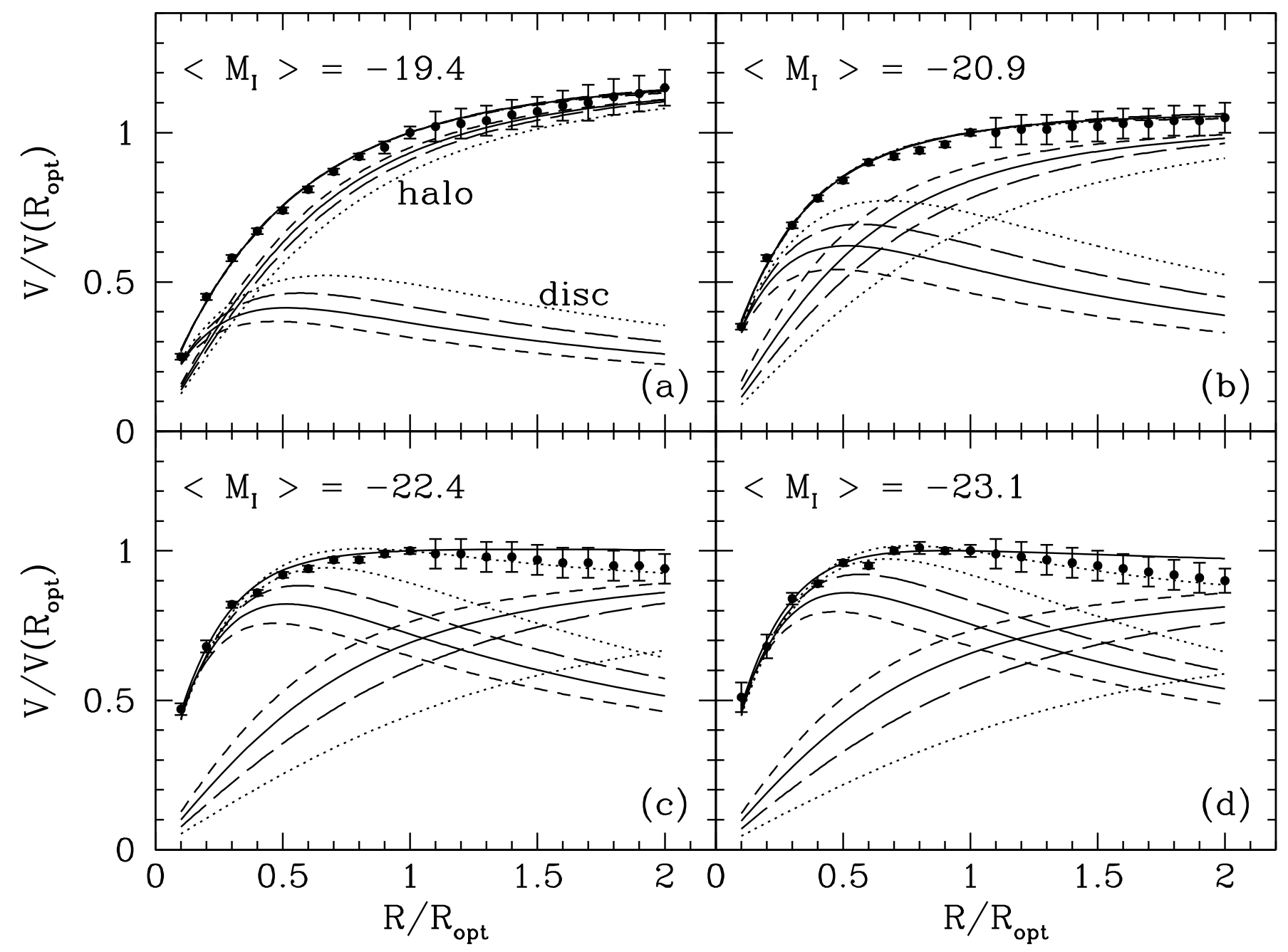

Fig. 8. Disc/halo decomposition of the co-added rotation curves of PSS96 for different galaxy luminosities; the disc RC, the dark halo contributions, and the total RC are shown for each choice of VML (or CML) disc RC. Line symbols are as in Fig. 7; the dotted lines represents the PSS96 mass model with the CML disc curve. In panels c), d)) the total (disc+halo) RCs of the three VML disc decompositions overlap.

iv) For RCs of low luminosity objects $\left(M_{I}<-21\right)$, we have $\mathrm{d} \log V /\left.\mathrm{d} \log R\right|_{2.2 h}>0.3$ (PSS96).

In the VML mass modelling, the same chain of arguments also applies, with only the difference that now the peak radius is at inner physical radii, i.e. at $\sim 1.7 \mathrm{~h}$. Noticeably, at this radius the observed RCs show an even steeper slope, d log $V /\left.\operatorname{dlog} R\right|_{1.7 h}>$ $\mathrm{d} \log V /\left.\mathrm{d} \log R\right|_{2.2 h}$, and therefore the discrepancy is even larger than in the CML case. For instance, for objects of $M_{I} \sim-21$, the observed $\operatorname{d} \log V /\left.\operatorname{d} \log R\right|_{2.2}{ }_{h} \sim 0.2$ : their RCs at $2.2 h$ can be, in the CML scenario, marginally reproduced by a NFW halo. But in the VML case where the disc contribution peaks, we have $\operatorname{dlog} V /\left.\operatorname{dlog} R\right|_{1.7 h} \simeq 0.25$, inconsistent with the NFW halo.

\section{Summary and conclusions}

We have investigated the radial profile of the stellar mass-to-light ratio in spiral galaxies, and seen how their inclusion changes the picture of the mass models of spiral galaxies. We derived the radial variations of $M_{\star} / L$ in galactic discs, as predicted by chemophotometric models in the "inside-out" formation scenario and as suggested by the observed colour gradients of spiral galaxies. The differential radial variation in $M_{\star} / L$ we predict for a "typical average" spiral turns out to be largely independent of the assumed SF history, stellar IMF, global galaxy colour, and other modelling details. It follows a profile of the type

$\frac{\frac{M_{\star}}{L_{I}}(R)}{\frac{M_{\star}}{L_{I}}\left(h_{I}\right)}=\exp \left\{-a(s)\left[\left(\frac{R}{h_{I}}\right)^{s}-1\right]\right\}$

with $0.5 \leq s \leq 1.1$ and $a(s)$ given in Eq. (3b). The predicted radial $M_{\star} / L$ variation can be significant, for instance in the $I$ band, $M_{\star} / L_{I}$ can decrease by up to a factor of two between 1 scalelength and the outskirts of the stellar disc $(4 h)$. We provide the corresponding variable mass-to-light rotation curve $V_{\mathrm{VML}}(R)$, that describes our best guess of the stellar disc contribution to the circular velocity in a "typical average" spiral galaxy. Since small variations $(\sim 20 \%)$ in the latter quantity can also affect the mass modelling, VML effects should not be neglected in objects showing radial colour gradients.

The resulting disc circular velocity $V_{\mathrm{VML}}(R)$ has some interesting features with respect to the classic constant mass-tolight (CML) Freeman curve. Since $M_{\star} / L$ decreases outwards, $V_{\mathrm{VML}}(R)$ peaks at smaller radii: at $1.5-1.9 h_{I}$ (rather than at $2.2 \mathrm{~h}$ ), and it approaches the Keplerian fall-off earlier, at 3-3.5 $h_{I}$ (rather than at $>4 h$ ). For a given observed RC, the steeper decline of the VML stellar contribution at the edge of the optical 
disc, with respect to the CML case, implies there an even steeper radial rise in the halo contribution.

With the average VML RC of stellar discs (combined with a cored dark matter halo), we have successfully fitted the co-added rotation curves of spiral galaxies of PSS96. The radial variations in the stellar $M_{\star} / L$ predicted by chemo-photometric models seem compatible with presently available kinematics (Fig. 8). Independent support may come from Salucci et al. (2008), who compared photometric and CML kinematic estimates of disc masses: despite the generally good agreement (the rms and the offset being only of 0.2 dex and 0.1 dex respectively), there were a few cases of photometric masses higher than the kinematic ones. These discrepancies could stem from VML discs.

The VML disc scenario may be very relevant in two open issues of galaxy structure. Firstly, we have shown that the radial variations of $M_{\star} / L$ allowed by chemo-photometric arguments, cannot reconcile the DM halo density profiles derived by mass modelling with those emerging from $\Lambda \mathrm{CDM}$ simulations. In contrast, the "standard" assumption of constant $M_{\star} / L$ helps NFW haloes to fit the observed kinematics. Because the colours of spiral galaxies typically redden inwards, the corresponding $M_{\star} / L$ variations are an additional problem for NFW profiles.

Secondly, we discuss whether, inside the optical disc, the stars alone, without any dark component, could account for the observed RCs via a radial variation of $M_{\star} / L$. This question was raised in the $80 \mathrm{~s}$, when evidence for DM on galactic scales started to gather. It is usually bypassed in view of the existence of non Keplerian, very extended HI RCs implying a huge mass discrepancy beyond the optical region. Nevertheless, the issue of the RC within the optical region is far from trivial, as no-DM scenarios like MOND and $f(R)$ theories of gravity are becoming popular (e.g. Sanders \& McGaugh 2002).

Figure 9 shows the co-added RC of galaxies with $\left\langle\mathrm{M}_{I}\right\rangle=$ -19.4 out to $3.5 h_{I}$. It has a steep slope which implies a clear mass discrepancy from $R=h_{I}$ outwards. We recall that at these luminosities the HI disc is dynamically important only outside the region we are considering here (de Blok et al. 2008). In Fig. 9 we show that the co-added curve can be reproduced well without any DM, with just a VML stellar disc. To reach this, however, we must invoke an increase in $M_{\star} / L$ with radius at the pace of $1+0.35\left(R / h_{I}\right)^{2}$. The corresponding colour variation (PST04) is a reddening of over $1 \mathrm{mag}$ in $(B-I)$ between $R=0.5 h_{I}$ and $3 h_{I}$. This is totally at odds with the observed radial bluing of about 0.4 mag over the same radial range (Fig. 3). We conclude that a dark component must also be present inside the luminous regions of disc galaxies.

The existence of VML discs may play an important role in the mass modelling within the MOND paradigm, where the observed distribution of the ordinary matter, stars, and HI gas is argued to completely account for the observed kinematics. In MOND, the circular velocity due to the distribution of the baryonic matter is "boosted" with respect to what we would have in Newtonian dynamics. From about 1 scalelength outward, a MOND self-gravitating Freeman disc implies a rotation curve constant with radius and with an amplitude that, at $R_{\mathrm{opt}}$, is about $20 \%$ more than in the Newtonian case. We consider highluminosity spiral galaxies with $V_{\mathrm{opt}} \sim 250 \mathrm{~km} \mathrm{~s}^{-1}, M_{I} \sim-23.5$, $h_{I} \sim 5.5 \mathrm{kpc}$, belonging to the second highest velocity bin of the co-added curves in PSS96 and shown in Fig. 10. In these objects the dynamical contribution of the HI disc and the stellar bulge are negligible in the region between 2 and 3 disc scalelengths. For a VML disc the stellar surface density is given by Eq. (5). We model the RC of a VML disc in MOND dynamics with $s=1$ (shallow profile) or $s=0.5$ (concave profile). Let us recall that

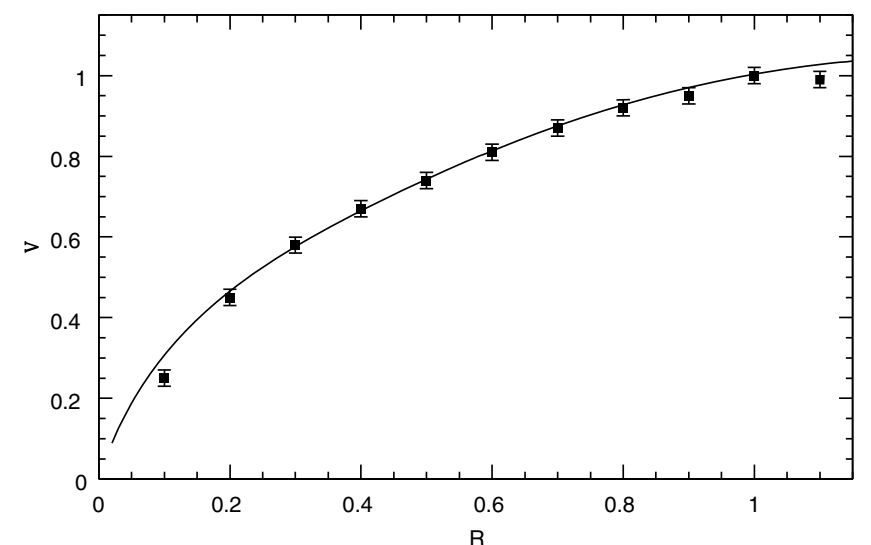

Fig. 9. No DM, VML model of the co-added RC at $M_{I}=-19.4$. The radial coordinate is in units of $R_{\mathrm{opt}}=3.2 h_{I}$.

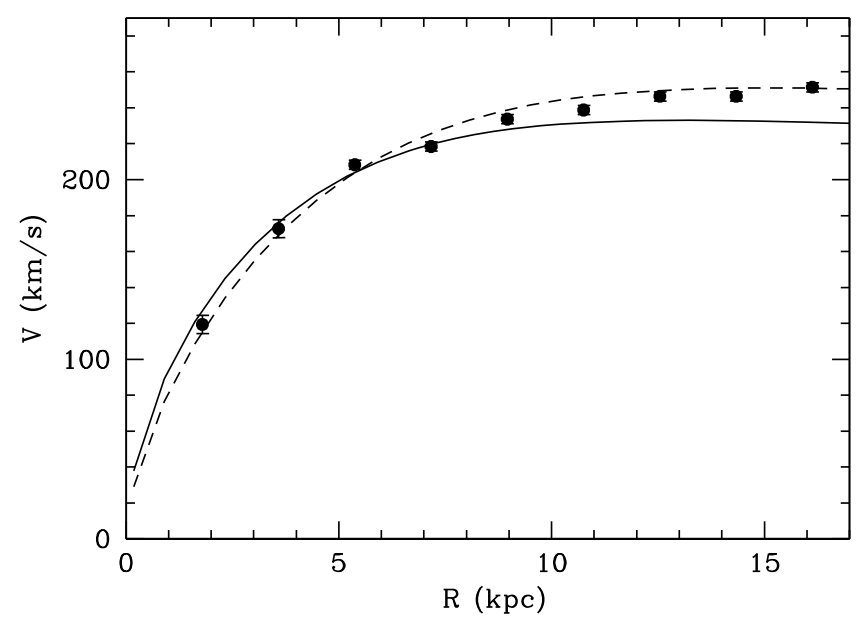

Fig. 10. MOND VML mass model of a co-added rotation curves of luminous objects. $s=1$ (dashed) and $s=0.5$ (solid) cases.

in Newtonian gravity both VML discs, or a CML disc, can fit this same RC when combined with a suitable cored dark halo (Fig. 8d). In MOND, instead, the outcome is different: while the $s=1 \mathrm{VML}$ disc accounts for the RC, the $s=0.5$ case does not (Fig. 10). This result is not a test of MOND, since we do not know the average $s$ in spiral galaxies, but indicates that in alternative theories to dark matter, the effects of $M_{\star} / L$ variations are much greater and potentially testable.

In the disc+dark halo mass modelling, the CML and VML discs yield equally good fits to the observed RCs (Fig. 8), so that one cannot use the RC to significantly reveal VML features. This is a non-trivial result: it is due not to an intrinsic degeneracy of the mass modelling, but to the fact that the allowed $M_{\star} / L$ variations are not very large. In the "average" late type spiral galaxy, chemo-photometric arguments indicate a decrease in $M_{\star} / L_{I}$ by a factor of 2 over the optical region, which is hardly distinguished from the kinematics. In the simplest VML disc case ( $s=1$ in Eq. (5)), for instance, the surface density distribution remains exponential, with just a scalelength about $20 \%$ shorter than that of the I-band luminosity profile.

Noticeably, in alternative scenarios to DM where the kinematics are completely determined by the distribution of the baryonic matter, the mild radial $M_{\star} / L$ gradients we predict are expected to play a relevant role.

Although the predicted $M_{\star} / L$ gradients do not radically alter the main results of traditional mass modelling, our comparison 
of the VML and CML assumptions shows that VML discs have an impact on RC decomposition: the trend is to obtain smaller disc normalization and smaller, but non-negligible, dark halo cores. The NFW halo profile becomes even less compatible with the observed kinematics in the inner regions; therefore, $M_{\star} / L$ gradients should in general not be neglected in mass modelling, and a combined chemo-photometric and dynamical analysis of individual objects (especially those showing strong colour gradients) is the best approach.

More ambitious, but not beyond reach, is the idea of using detailed kinematic analysis as an independent test of the predictions of chemo-photometric models. By turning the above argument around and by assuming a complete inner baryon dominance in the innermost regions, one can possibly aim at measuring the variations in the stellar $M_{\star} / L$ directly from high-quality rotation curves and check the predictions of chemo-photometric models. Also, since the prediction is that the scalelength of the stellar density profile is shorter than the luminous scalelength (by at least $20 \%, s=1$ case), future investigations could aim at revealing VML discs by estimating the dynamical scalelengths from the observed RCs and then comparing them with the photometric ones.

Acknowledgements. We thank the anonymous referee for many detailed comments that helped us improve the presentation. We are grateful to Chris Flynn for careful reading and useful remarks on the manuscript. L.P. acknowledges kind hospitality from SISSA and from the Department of Astronomy in Padua on various visits. This study was financed by the Academy of Finland (grant no. 208792), by the 6th Framework Programme of the European Commission (Marie Curie Action no. MEIF-CT-2005-010884) and by the Italian MIUR. We used the VizieR On-line Data Catalogue to retrieve observational data used in this paper.

\section{References}

Ashman, K. M., Salucci, P., \& Persic, M. 1993, MNRAS, 260, 610

Bakos, J., Trujillo, I., \& Pohlen, M. 2008, ApJ, 683, L103

Bell, E. F., \& Bower, R. G. 2000a, MNRAS, 319, 235

Bell, E. F., \& de Jong, R. S. 2000b, MNRAS, 312, 497

Bell, E. F., \& de Jong, R. S. 2001, ApJ, 550, 212

de Blok, W. J. G. 2007, in Island Universes. Structure and evolution of disc galaxies, ed. R. S. de Jong (ASSP, Springer), 89

de Blok, W.J.G., \& Bosma, A. 2002, A\&A, 385, 816

de Blok, W. J. G., Walter, F., Brinks, E. 2008, AJ, 136, 2648

Boissier, S., \& Prantzos, N. 1999, MNRAS, 307, 857

Boissier, S., \& Prantzos, N. 2000, MNRAS, 312, 398

Boissier, S., \& Prantzos, N. 2001, MNRAS, 325, 321

Burkert, A. 1995, ApJ, 447, L25

Burkert, A., Truran, J. W., \& Hensler, G. 1992, ApJ, 391, 651

Chabrier, G. 2001, ApJ, 554, 1274

Chabrier, G. 2002, ApJ, 567, 304

Chiappini, C., Matteucci, F. M., \& Gratton, R. 1997, ApJ, 477, 765

Chiappini, C., Matteucci, F., \& Romano, D. 2001, ApJ, 554, 1044

Dopita, M. A., \& Ryder, S. D. 1994, ApJ, 430, 163

Flynn, C., Holmberg, J., Portinari, L., Fuchs, B., \& Jahreiß, H. 2006, MNRAS, 372, 1149

Freeman, K. 1970, ApJ, 160, 811

Garnett, D., Shields, G., Skillman, E., Sagan, S., \& Dufour, R. 1997, ApJ, 489, 63

Gentile, G., Salucci, P., Klein, U., Vergani, D., \& Kalberla, P. 2004, MNRAS, 351903
Gentile, G., Burkert, A., Salucci, P., Klein, U., \& Walter, F. 2005, ApJ, 634, L145 Gummersbach, C. A., Kaufer, A., Schäfer, D. R., Szeifert, T., \& Wolf, B. 1998, A\&A, 338, 881

Jansen, R. A., Franx, M., Fabricant, D., et al. 2000, ApJS, 126, 271

de Jong, R. S. 1996a, A\&AS, 118, 557

de Jong, R. S. 1996b, A\&A, 313, 45

de Jong, R. S. 1996c, A\&A, 313, 377

de Jong, R. S., \& van der Kruit, P. C. 1994, A\&AS, 106, 451

Kassin, S., de Jong, R. S., \& Weiner, B. J. 2006, ApJ, 643, 804

Kennicutt, R. C. 1983, ApJ, 272, 54

Kennicutt, R. C., Tamblyn, P., \& Congdon, C. E. 1994, ApJ, 435, 22

Kent, S. M. 1986, AJ, 91, 1301

Kranz, T., Slyz, A., \& Rix, H.-W. 2003, ApJ, 586, 143

Kroupa, P. 1998, ASP Conf. Ser., 134, 483

Kuzio de Naray, R., McGaugh, S. S., de Blok, W. J. G., et al. 2006, ApJS, 165, 461

Larson, R. B. 1976, MNRAS, 176, 31

Larson, R. B. 1998, MNRAS, 301, 569

López-Corredoira, M., Cabrera-Lavers, A., \& Mahoney, T. J. 2007, AJ, 133, 2007

Lynden-Bell, D. 1975, Vistas in Astr., 19, 229

Martin, P., \& Roy, J. R. 1994, ApJ, 424, 599

Matteucci, F., \& François, P. 1989, MNRAS, 239, 885

Mollá, M., \& Diaz, A. I. 2005, MNRAS, 358, 521

Mollá, M., Ferrini, F., \& Diaz, A. I. 1996, ApJ, 466, 668

Mollá, M., Ferrini, F., \& Diaz, A. I. 1997, ApJ, 475, 519

Navarro, J. F., Frenk, C. S., \& White, S. D. M. 1996, ApJ, 462, 563

Navarro, J. F., Frenk, C. S., \& White, S. D. M. 1997, ApJ, 490, 493

Pagel, B. E. J. 1997, Nucleosynthesis and Chemical Evolution of Galaxies, Cambridge University Press

Persic, M., \& Salucci, P. 1988, MNRAS, 234, 131

Persic, M., Salucci, P., \& Stel, F. 1996, MNRAS, 281, 27 (PSS96)

Portinari, L., \& Chiosi, C. 1999, A\&A, 350, 827

Portinari, L., \& Chiosi, C. 2000, A\&A, 355, 929

Portinari, L., Chiosi, C., \& Bressan, A. 1998, A\&A, 334, 505

Portinari, L., Sommer-Larsen, J., \& Tantalo, R. 2004, MNRAS, 347, 691 (PST04)

Prantzos, N., \& Boissier, S. 2000, MNRAS, 313, 338

Press, W. H., Teukolsky, S. A., Vetterling, W. T., et al. 1992, Numerical Recipes (Cambridge University Press)

Renda, A., Kawata, D., Fenner, Y., et al. 2005, MNRAS, 356, 1071

Roberts, M. S., \& Haynes, M. P. 1994, ARA\&A, 32, 115

Roškar, R., De Battista, V. P., Quinn, T. R., Stinson, G. S., \& Wadsley, J. 2008, ApJ, 684, L79

Ryder, S. D., \& Dopita, M. A. 1994, ApJ, 430, 142

Salpeter, E. E. 1955, ApJ, 121, 161

Salucci, P. 2001, MNRAS, 320, L1

Salucci, P., Walter, F., \& Borriello, A. 2003, A\&A, 409, 53

Salucci, P., Lapi, A., Tonini, C., et al. 2007, MNRAS, 378, 41

Salucci, P., Yegorova, I., \& Drory, N. 2008, MNRAS, 388, 159

Sanders, R. H., \& McGaugh, S. S. 2002, ARA\&A, 40, 263

Schönrich, R., \& Binney, J. 2009, MNRAS, 396, 203

Shaver, P. A., McGee, R. X., Newton, L. M., Danks, A. C., \& Pottasch, S. R. 1983, MNRAS, 204, 53

Smartt, S. J., \& Rolleston, W. R. J. 1997, ApJ, 481, L47

Sommer-Larsen, J. 1991, MNRAS, 250, 356

Sommer-Larsen, J., Götz, M., \& Portinari, L. 2003, ApJ, 596, 47

Spano, M., Marcelin, M., Amram, P., et al. 2008, MNRAS, 383, 297

Spekkens, K., \& Giovanelli, R. 2006, AJ, 132, 1426

Tinsley, B. 1980, Fund. Cosmic Phys. 5, 287

Treuthardt, P., Salo, H., \& Buta, R. 2009, AJ, 137, 19

Verheijen, M. A. W. 1997, Ph.D. Thesis, (The Netherlands: University of Groningen)

van Zee, L., Salzer, J. J., Haynes, M. P., O’Donoghue, A. A., \& Balonek, T. J. 1998, AJ, 116, 2805 